\title{
Gap continuity of multimaps
}

\author{
Jean-Paul Penot*
}

\begin{abstract}
We introduce a notion of continuity for multimaps (or set-valued maps) which is mild. It encompasses both lower semicontinuity and upper semicontinuity. We give characterizations and we consider some permanence properties. This notion can be used for various purposes. In particular, it is used for continuity properties of subdifferentials and of value functions in parametrized optimization problems. We also prove an approximate selection theorem.

Mathematics Subject Classifications (2000): 47H04, 54C60, 54C65, $26 \mathrm{~B} 05$.

Keywords: correspondence, gap-continuity, lower semicontinuity, multimap, multifunction, multimapping, set-valued map, subdifferential, semicontinuity, value function.
\end{abstract}

\section{Introduction}

It is the purpose of this note to present a notion of continuity for multimaps (or multimappings, or multifunctions, or correspondences or set-valued maps) called gap continuity. It relies on the notion of gap between two sets. It is rather coarse, but natural: roughly speaking, one has that a multimap $F: X \rightrightarrows Y$ between a topological space and a metric space is gap-continuous at some point $\bar{x}$ of $X$ if $F(x)$ and $F(\bar{x})$ are not too far apart when $x$ is sufficiently close to $\bar{x}$. Precise characterizations are presented in section 2. Because this notion is not as restrictive as usual lower semicontinuity or upper semicontinuity, it is more likely to be satisfied by concrete examples and it is more versatile. As an example, we note that a constraint qualification condition is formulated in terms of gap in [6, Prop. 2.5].

In spite of its weakness, gap-continuity enjoys useful properties. Stability properties are displayed in section 3. In particular, one obtains that if $F: X \rightrightarrows Y$ is upper semicontinuous and $G: X \rightrightarrows Z$ is lower semicontinuous, then $H:=(F, G): X \rightrightarrows Y \times Z$ is gap-continuous. Section 4 is devoted to an application to parametrized optimization and to criteria ensuring gap continuity of subdifferentials of convex functions. Such multimaps are not lower semicontinuous, unless they are single-valued, as shown by Gossez ([10]). These criteria are used in [1].

\footnotetext{
*Faculté des Sciences, Mathématiques UMR CNRS 5142, B.P. 1155, 64013 PAU Cedex, France
} 


\section{Definitions and characterizations}

Our study relies on the following simple concept which has been used for some time, especially for questions of convergence (see [4], [16], for instance). It is sometime called the distance between the two sets and denoted by $D(A, B)$ or $d(A, B)$; these choices may be confusing since this notion is not a metric ( the triangular inequality is not satisfied with this notion).

Definition 2.1 The gap between two subsets $A, B$ of a metric space $(Y, d)$ is given by

$$
\operatorname{gap}(A, B)=\inf \{d(a, b): a \in A, b \in B\}
$$

with the usual convention: $\operatorname{gap}(A, B)=\infty$ if $A=\varnothing$ or $B=\varnothing$.

This concept satisfies natural properties. In particular, if for $i:=1, \ldots, m, A_{i}$ and $B_{i}$ are subsets of a metric space $\left(X_{i}, d_{i}\right)$ then

$$
\operatorname{gap}\left(A_{1} \times \ldots \times A_{m}, B_{1} \times \ldots \times B_{m}\right)=\max _{1 \leq i \leq m} \operatorname{gap}\left(A_{i}, B_{i}\right)
$$

when $X:=X_{1} \times \ldots \times X_{m}$ is endowed with the metric $d_{\infty}$ given by $d_{\infty}\left(\left(x_{i}\right),\left(y_{i}\right)\right):=\max d_{i}\left(x_{i}, y_{i}\right)$, while if $X$ is endowed with the metric $d$ given by $d\left(\left(x_{i}\right),\left(y_{i}\right)\right):=d_{1}\left(x_{1}, y_{1}\right)+\ldots+d_{m}\left(x_{m}, y_{m}\right)$ one has

$$
\operatorname{gap}\left(A_{1} \times \ldots \times A_{m}, B_{1} \times \ldots \times B_{m}\right)=\sum_{i=1}^{m} \operatorname{gap}\left(A_{i}, B_{i}\right),
$$

as easily checked. Moreover, if $X$ is a normed vector space and $A, A^{\prime}, B, B^{\prime}, A_{i}, B_{i}(i=1, \ldots, m)$ are subsets of $X$ one has

$$
\begin{aligned}
\operatorname{gap}\left(A+A^{\prime}, B+B^{\prime}\right) & \leq \operatorname{gap}(A, B)+\operatorname{gap}\left(A^{\prime}, B^{\prime}\right) . \\
\left.\operatorname{gap}\left(\bigcup_{i=1}^{m} A_{i}\right), \bigcup_{i=1}^{m} B_{i}\right) & \leq \min _{i=1, \ldots, m} \operatorname{gap}\left(A_{i}, B_{i}\right), \\
\operatorname{gap}\left(\operatorname{co}\left(\bigcup_{i=1}^{m} A_{i}\right), \operatorname{co}\left(\bigcup_{i=1}^{m} B_{i}\right)\right) & \leq \min _{i=1, \ldots, m} \operatorname{gap}\left(A_{i}, B_{i}\right),
\end{aligned}
$$

where $A+A^{\prime}:=\left\{a+a^{\prime}: a \in A, a^{\prime} \in A^{\prime}\right\}$ and $\operatorname{co}(A)$ stands for the convex hull of $A$.

The following statement shows the usefulness of the notion of gap in convex analysis.

Proposition 2.1 Let $A$ and $B$ be two nonempty convex subsets of a normed vector space $X$ such that $\operatorname{gap}(A, B)>0$. Then there exist $c \in \mathbb{R}$ and $x^{*} \in X^{*} \backslash\{0\}$ such that

$$
\sup _{a \in A}\left\langle x^{*}, a\right\rangle<c<\inf _{b \in B}\left\langle x^{*}, b\right\rangle .
$$


Proof. Let $r:=\operatorname{gap}(A, B)$. Then the open ball $B(0, r)$ with center 0 and radius $r$ does not meet $A-B:=A+(-B)$ which is convex. Applying the Hahn-Banach theorem, we find some $x^{*} \in X^{*} \backslash\{0\}$ such that $\left\langle x^{*}, a-b\right\rangle \leq \inf \left\{\left\langle x^{*}, x\right\rangle: x \in B(0, r)\right\}=-r\left\|x^{*}\right\|<0$. The result follows by taking $c:=\sup \left\{\left\langle x^{*}, a\right\rangle: a \in A\right\}+r^{\prime}\left\|x^{*}\right\|$ with $\left.r^{\prime} \in\right] 0, r[$.

The following continuity condition is quite mild. Nonetheless, it plays a key role in the companion paper [1]. It also deserves some attention for its independent interest, as we intend to show below.

Definition 2.2 A multimap $F: X \rightrightarrows Y$ between a topological space $X$ and a metric space $Y$ is said to be gap-continuous at $\bar{x} \in X$ if

$$
\operatorname{gap}(F(\bar{x}), F(x)) \rightarrow 0 \text { as } x \rightarrow \bar{x} .
$$

We supplement this definition with a variant which is slightly more demanding.

Definition 2.3 A multimap $F: X \rightrightarrows Y$ between a topological space and a metric space is said to be circa-gap-continuous at $\bar{x} \in X$ if

$$
\operatorname{gap}\left(F(x), F\left(x^{\prime}\right)\right) \rightarrow 0 \text { as } x, x^{\prime} \rightarrow \bar{x} .
$$

Clearly, when $F$ is single-valued, gap continuity and circa-gap-continuity coincide with continuity. Since the gap does not satisfy the triangular inequality, the preceding two notions do not coincide.

Example 1. Let $F: \mathbb{R} \rightrightarrows \mathbb{R}$ be defined by $F(0):=[-1,+1], F(x):=\left\{|x|^{-1} x\right\}$ for $x \in \mathbb{R} \backslash\{0\}$. Then $F$ is gap-continuous at 0 , but it is not circa-gap-continuous at 0 since $\operatorname{gap}\left(F(x), F\left(x^{\prime}\right)\right)=2$ for $x>0, x^{\prime}<0$.

Example 2. Let $f: \mathbb{R} \rightarrow \mathbb{R}$ and let $F: \mathbb{R} \rightrightarrows \mathbb{R}$ be defined by $F(\bar{x}):=f(\bar{x})-\mathbb{R}_{+}, F(x):=$ $f(x)+\mathbb{R}_{+}$for $x \in \mathbb{R} \backslash\{\bar{x}\}$. Then $F$ is gap-continuous at $\bar{x}$ if, and only if $f$ is upper semicontinuous at $\bar{x}$. A corresponding characterization of the lower semicontinuity of $f$ can be given.

The following examples dealing with a classical notion in the geometry of Banach spaces will be generalized in section 4 .

Example 3. Let $F: \mathbb{R}^{d} \rightrightarrows \mathbb{R}^{d}$ be the duality mapping associated with one of the usual norms $\left(\|\cdot\|_{1},\|\cdot\|_{p},\|\cdot\|_{\infty}\right)$ of $\mathbb{R}^{d}:$ it is given by $F(x):=\left\{y \in \mathbb{R}^{d}: x \cdot y=\|x\|^{2},\|y\|=\|x\|\right\}=\frac{1}{2} \partial\|\cdot\|^{2}(x)$. Then, $F$ is gap-continuous at each point of $\mathbb{R}^{d}$ (see also Proposition 4.1 below).

Example 4. More generally, let $X$ be a Banach space whose dual $X^{*}$ satisfies the weak* Kadec-Klee property in the following sense: any bounded sequence $\left(x_{n}^{*}\right)$ which has a weak* cluster point $x^{*}$ satisfying $\left(\left\|x_{n}^{*}\right\|\right) \rightarrow\left\|x^{*}\right\|$ has a subnet which converges in norm to $x^{*}$. Then the duality mapping $J: X \rightrightarrows X^{*}$ given by

$$
J(x):=\left\{x^{*} \in X^{*}:\left\langle x^{*}, x\right\rangle=\|x\|^{2},\left\|x^{*}\right\|=\|x\|\right\}
$$

is gap-continuous at each point $\bar{x} \in X$. In fact, otherwise there exist $\alpha>0$ and a sequence $\left(x_{n}\right) \rightarrow \bar{x}$ such that $\left\|x_{n}^{*}-\bar{x}^{*}\right\| \geq \alpha$ for every $n \in \mathbb{N}, \bar{x}^{*} \in J(\bar{x}), x_{n}^{*} \in J\left(x_{n}\right)$; then, since $J$ has nonempty values and is locally bounded, for any choice of a sequence $\left(x_{n}^{*}\right)$ satisfying $x_{n}^{*} \in J\left(x_{n}\right)$ 
for all $n$, and for any weak* cluster point $x^{*}$ of $\left(x_{n}^{*}\right)$, one has, for some subnet $\left(x_{n(i)}, x_{n(i)}^{*}\right)_{i \in I}$ of $\left(x_{n}, x_{n}^{*}\right)$

$$
\left\|x^{*}\right\| \leq \liminf _{n}\left\|x_{n}^{*}\right\|, \quad\left\langle x^{*}, \bar{x}\right\rangle=\lim _{i \in I}\left\langle x_{n(i)}^{*}, x_{n(i)}\right\rangle=\lim _{i \in I}\left\|x_{n(i)}\right\|^{2}=\|\bar{x}\|^{2},
$$

hence $\left\|x^{*}\right\|=\lim _{n}\left\|x_{n}^{*}\right\|$ since $\left\|x_{n}^{*}\right\|=\left\|x_{n}\right\|=\|\bar{x}\|$. Thus $\left(x_{n}^{*}\right)$ has a subnet which norm converges to $x^{*}$; since $\left\langle x^{*}, \bar{x}\right\rangle=\|\bar{x}\|^{2}$ and $\left\|x^{*}\right\|=\|\bar{x}\|$, one has $x^{*} \in J(\bar{x})$, a contradiction with $\left\|x_{n}^{*}-\bar{x}^{*}\right\| \geq \alpha$ for every $n \in \mathbb{N}$. Note that Hilbert spaces and $L_{p}$ spaces satisfy the weak ${ }^{*}$ Kadec-Klee property. Moreover, any Banach space with a separable dual space can be given an equivalent norm satisfying the weak* Kadec-Klee property (see [12, p. 220]).

Let us compare these notions to familar continuity concepts. The proofs of these comparisons are immediate.

Lemma 2.1 Let $F: X \rightrightarrows Y$ be a multifunction between a topological space and a metric space.

(a) If $F$ is lower semicontinuous (l.s.c.) at $(\bar{x}, \bar{y}) \in \operatorname{graph} F$ in the sense that $d(\bar{y}, F(x)) \rightarrow 0$ as $x \rightarrow \bar{x}$, then $F$ is circa-gap-continuous at $\bar{x}$, hence is gap-continuous at $\bar{x}$. Conversely, if $F(\bar{x})$ is a singleton $\{\bar{y}\}$ and if $F$ is gap-continuous at $\bar{x}$, then $F$ is l.s.c. at $(\bar{x}, \bar{y})$.

(b) If $F$ is Hausdorff upper semicontinuous (H.u.s.c.) at $\bar{x} \in \operatorname{int} \operatorname{dom} F$ in the sense that $\sup \{d(y, F(\bar{x})): y \in F(x)\} \rightarrow 0$ as $x \rightarrow \bar{x}$, in particular if $F$ is upper semicontinuous (u.s.c.) at $\bar{x}$, then $F$ is gap-continuous at $\bar{x}$. The converse holds if for $x \neq \bar{x}$ close to $\bar{x}$ the set $F(x)$ is a singleton.

The converses requiring strong assumptions, we see that gap-continuity is a weak notion of semicontinuity. The following simple counterexamples show that gap-continuity is strictly weaker than lower semicontinuity and upper semicontinuity.

Example 5. Given $c: X \rightarrow Y$, where $X$ is a topological space and $Y$ is a normed vector space, and $r: X \rightarrow \mathbb{R}_{+}$, let $F: X \rightrightarrows Y$ be given by $F(x):=c(x)+r(x) B$, where $B$ is the closed unit ball of $Y$. If for some $\bar{x} \in X$ one has $\|c(x)-c(\bar{x})\| \leq r(x)+r(\bar{x})$ for $x$ close to $\bar{x}$, then $F$ is gap-continuous at $\bar{x}$. If $c$ or $r$ are discontinuous at $\bar{x}$, lower or upper semicontinuity of $F$ is not necessarily ensured.

Example 6. Let $X=Y:=\mathbb{R}$ and let $F: X \rightrightarrows Y$ be given by $F(x):=\{0\}$ for $x<0$, $F(0):=(-1,1), F(x):=\{-1,1\}$ for $x>0$. Then $F$ is gap-continuous on $X$, but $F$ is neither lower semicontinuous nor upper semicontinuous at 0 .

Example 7. Let $X=Y:=\mathbb{R}$ and let $F: X \rightrightarrows Y$ be given by $F(x):=\{-1,2\}$ for $x<0$, $F(0):=[-1,1], F(x):=\{1,2\}$ for $x>0$. Then $F$ is circa-gap-continuous on $X$, but $F$ is neither lower semicontinuous nor upper semicontinuous at 0 .

Example 8. Let $X$ be a topological space, let $Y$ be a normed vector space with dual spaces $Y^{*}$ and let $f: X \rightarrow Y^{*}, r: X \rightarrow \mathbb{R}$ be continuous with $f(\bar{x}) \neq 0$ for some $\bar{x} \in X$. Then $F: X \rightrightarrows Y$ given by $F(x):=\{y \in Y:\langle f(x), y\rangle=r(x)\}$ is gap continuous at $\bar{x}$ and graph closed, but it is not upper semicontinuous.

Let us note the following obvious reformulation.

Lemma 2.2 For a multimap $F: X \rightrightarrows Y$ between a topological space $X$ and a metric space $Y$ one has the implications $(d) \Rightarrow(c) \Rightarrow(b) \Rightarrow(a)$ among the following assertions. If $X$ is a metric 
space, or more generally, if $\bar{x} \in X$ has a countable base of neighborhoods or if there exists a function $h: X \rightarrow \mathbb{R}$ continuous at $\bar{x}$ with $h(\bar{x})=0, h(x)>0$ for $x \neq \bar{x}$, these assertions are equivalent:

(a) $F$ is gap-continuous at $\bar{x}$;

(b) for any $\varepsilon>0$ there exists a neighborhood $V$ of $\bar{x}$ such that for any $v \in V$ one can find $y(v) \in F(v)$ such that $d(y(v), F(\bar{x}))<\varepsilon$;

(c) there exist a neighborhood $U$ of $\bar{x}$ and $f: U \rightarrow Y$ such that $f(u) \in F(u)$ for all $u \in U$ and $d(f(u), F(\bar{x})) \rightarrow 0$ as $u \rightarrow \bar{x}$;

(d) there exist a neighborhood $U$ of $\bar{x}$ and $f: U \rightarrow Y, g: U \rightarrow Y$ such that $f(u) \in F(u)$, $g(u) \in F(\bar{x})$ for all $u \in U$ and $d(f(u), g(u)) \rightarrow 0$ as $u \rightarrow \bar{x}$.

In particular, when $Y$ is a n.v.s., $F$ is gap-continuous at $\bar{x}$ iff

$$
\forall \varepsilon>0 \exists \delta>0: \forall x \in B(\bar{x}, \delta) \quad F(x) \cap\left(F(\bar{x})+\varepsilon B_{Y}\right) \neq \varnothing .
$$

Proof. The implications $(\mathrm{d}) \Rightarrow(\mathrm{c}) \Rightarrow(\mathrm{b}) \Rightarrow(\mathrm{a})$ are obvious. Let us show that $(\mathrm{a}) \Rightarrow(\mathrm{d})$ when there exists a function $h: X \rightarrow \mathbb{R}$ continuous at $\bar{x}$ with $h(\bar{x})=0, h(x)>0$ for $x \neq \bar{x}$. Setting

$$
\delta(x):=\operatorname{gap}(F(\bar{x}), F(x)),
$$

let $U$ be a neighborhood of $\bar{x}$ such that $\delta(x)<+\infty$ for $x \in U$. Then, for $u \in U$, the set $F(u)$ is nonempty (and thus $F(\bar{x})$ is nonempty too) and we can find some $y_{u} \in F(u), \bar{y}_{u} \in F(\bar{x})$ such that

$$
d\left(y_{u}, \bar{y}_{u}\right) \leq \delta(u)+h(u)
$$

as easily seen by considering separately the case $u=\bar{x}$ and the case $u \in U \backslash\{\bar{x}\}$. Then, setting $f(u):=y_{u}, g(u):=\bar{y}_{u}$, we get $d(f(u), g(u)) \leq \delta(u)+h(u) \rightarrow 0$ as $u \rightarrow \bar{x}$. When $X$ is endowed with a metric $d_{X}$ inducing its topology, we can define $h$ by $h(x):=d_{X}(u, \bar{x})$, while when $\bar{x}$ has a countable base of neighborhoods $\left(V_{n}\right)$, we may suppose $V_{0}:=X, V_{n+1} \subset V_{n}$ for all $n$ and define $h$ by $h(\bar{x})=0, h(x)=1 /(n+1)$ for $x \in V_{n} \backslash V_{n+1}$, so that $h(x) \rightarrow 0$ as $x \rightarrow \bar{x}$.

The following corollary is also a consequence of the fact pointed out to us by $\mathrm{C}$. Zalinescu that, for any two subsets $A, B$ of a normed vector space, $\operatorname{gap}(A, B)=d(0, A-B)$.

Corollary 2.1 If $X$ is a topological space and if $Y$ is a normed vector space, $F: X \rightrightarrows Y$ is gap-continuous at $\bar{x}$ if, and only if, the multimap $G: X \rightrightarrows Y$ given by $G(x):=F(x)-F(\bar{x})$ is lower semicontinuous at $(\bar{x}, 0)$ in the sense that $d(0, G(x)) \rightarrow 0$ as $x \rightarrow \bar{x}$.

Let us give a local version of Lemma 2.2 for circa-gap-continuity. It will lead to a global result.

Lemma 2.3 For a multimap $F: X \rightrightarrows Y$ between two metric spaces $X$ and $Y$ the following assertions are equivalent:

(a) $F$ is circa-gap-continuous at $\bar{x}$;

(b) for every $\varepsilon>0$ there exists a neighborhood $V$ of $\bar{x}$ such that for any $v, w \in V$ one can find $y(v, w) \in F(v)$ such that $d(y(v, w), y(w, v))<\varepsilon$;

(c) there exist a neighborhood $U$ of $\bar{x}$ and $f: U \times U \rightarrow Y$ such that $f(v, w) \in F(v)$ for all $v, w \in U$ and $d(f(v, w), f(w, v)) \rightarrow 0$ as $v, w \rightarrow \bar{x}$. 
Proof. Again, the implications $(\mathrm{c}) \Rightarrow(\mathrm{b}) \Rightarrow(\mathrm{a})$ are obvious. Thus, it suffices to show that (a) $\Rightarrow(\mathrm{c})$. For that purpose, using the gap-continuity of $F$ at $\bar{x}$, we pick a neighborhood $U$ of $\bar{x}$ such that for all $v \in U, F(v)$ is nonempty, we pick $f(v, v)$ arbitrary in $F(v)$. We endow $U$ with a total order (using the axiom of choice) and we set

$$
\gamma(v, w):=\operatorname{gap}(F(v), F(w)) .
$$

Then, for $(v, w) \in U^{2}$ with $v<w$ we pick $f(v, w) \in F(v), f(w, v) \in F(w)$ such that $d(f(v, w), f(w, v)) \leq \gamma(v, w)+d(v, w)$. Since the order is total, for all $(v, w) \in U^{2}$ we have either $v<w$ and the preceding inequality, or $w<v$ and $d(f(v, w), f(w, v))=d(f(w, v), f(v, w)) \leq$ $\gamma(w, v)+d(v, w)$. In both cases we have $d(f(v, w), f(w, v)) \rightarrow 0$ as $v, w \rightarrow \bar{x}$.

A global version can be given.

Proposition 2.2 For a multimap $F: X \rightrightarrows Y$ between a metric space $X$ and a normed vector space $Y$, the following assertions are equivalent when $F$ has convex images:

(a) $F$ is circa-gap-continuous on $X$;

(b) for every $x \in X$ and every $\varepsilon>0$ there exists a neighborhood $U$ of $x$ such that for every $u, v \in U$ one can find $y(u, v) \in F(u)$ such that $d(y(u, v), y(v, u))<\varepsilon$;

(c) for every $\varepsilon>0$ there exist a symmetric neighborhood $W_{\varepsilon}$ of the diagonal in $X \times X$ and $f_{\varepsilon}: W_{\varepsilon} \rightarrow Y$ such that $f_{\varepsilon}(u, v) \in F(u)$ and $d\left(f_{\varepsilon}(u, v), f_{\varepsilon}(v, u)\right)<\varepsilon$ for all $(u, v) \in W_{\varepsilon}$;

(d) there exist a symmetric neighborhood $W$ of the diagonal in $X \times X$ and $f: W \rightarrow Y$ such that $f(u, v) \in F(u)$ for all $(u, v) \in U$ and for all $\bar{x} \in X, d(f(u, v), f(v, u)) \rightarrow 0$ as $(u, v) \rightarrow(\bar{x}, \bar{x})$.

Proof. Again, it suffices to prove the implication $(\mathrm{a}) \Rightarrow(\mathrm{d})$. We set $\gamma(u, v):=\operatorname{gap}(F(u), F(v))$ and we take for $W$ the set of $(u, v) \in X^{2}$ such that $\gamma(u, v)<+\infty$. Endowing $X$ with a total order, for $(u, v) \in W$ with $u<v$, we pick $f(u, v) \in F(u), f(v, u) \in F(v)$ such that $d(f(u, v), f(v, u)) \leq \gamma(u, v)+d(u, v)$. For $u=v$, we take $f(u, v)$ arbitrarily in $F(u)$. Then, if $(u, v) \in W$ is such that $v<u$ we get $d(f(u, v), f(v, u))=d(f(v, u), f(u, v)) \leq \gamma(v, u)+d(v, u)=$ $\gamma(u, v)+d(u, v)$. In both cases we have $d(f(u, v), f(v, u)) \rightarrow 0$ as $(u, v) \rightarrow(\bar{x}, \bar{x})$.

Now let us give a characterization of gap-continuity in terms of support functions. Recall that the support function of a subset $C$ of some n.v.s. $Y$ is the function $\sigma_{C}: Y^{*} \rightarrow \overline{\mathbb{R}}:=$ $\mathbb{R} \cup\{-\infty,+\infty\}$ given by

$$
\sigma_{C}\left(y^{*}\right):=\sigma\left(y^{*}, C\right):=\sup \left\{\left\langle y^{*}, y\right\rangle: y \in C\right\} .
$$

Proposition 2.3 Let $F: X \rightrightarrows Y$ be a multimap from a topological space to a n.v.s. with nonempty closed convex values. Then $F$ is gap-continuous at $\bar{x} \in X$ if, and only if,

$$
\liminf _{x \rightarrow \bar{x}} \inf \left\{\sigma\left(y^{*}, F(x)\right)+\sigma\left(-y^{*}, F(\bar{x})\right): y^{*} \in Y^{*},\left\|y^{*}\right\|=1\right\} \geq 0 .
$$

Proof. Let us first show that the condition is necessary. Assume on the contrary that $F$ is gap-continuous at $\bar{x} \in X$ and there exist some $r>0$ and some net $\left(x_{i}\right)_{i \in I}$ with limit $\bar{x}$ such that for every $i \in I$ one has

$$
\inf \left\{\sigma\left(y^{*}, F\left(x_{i}\right)\right)+\sigma\left(-y^{*}, F(\bar{x})\right): y^{*} \in Y^{*},\left\|y^{*}\right\|=1\right\}<-r .
$$


Then for every $i \in I$ one can find some $y_{i}^{*} \in Y^{*}$ such that $\sigma\left(y_{i}^{*}, F\left(x_{i}\right)\right)+\sigma\left(-y_{i}^{*}, F(\bar{x})\right)<-r$, $\left\|y_{i}^{*}\right\|=1$. Thus, setting $s_{i}:=\sigma\left(y_{i}^{*}, F\left(x_{i}\right)\right)$, for $i \in I$ large enough we get that $s_{i}$ is finite since $F\left(x_{i}\right)$ is nonempty by the gap-continuity of $F$. For

$$
D_{i}:=\left\{y \in Y:\left\langle y_{i}^{*}, y\right\rangle \leq s_{i}\right\}, \quad E_{i}:=\left\{y \in Y:\left\langle y_{i}^{*}, y\right\rangle \geq s_{i}+r\right\}
$$

we have $F\left(x_{i}\right) \subset D_{i}, F(\bar{x}) \subset E_{i}$, hence $\operatorname{gap}\left(F\left(x_{i}\right), F(\bar{x})\right) \geq \operatorname{gap}\left(D_{i}, E_{i}\right) \geq r$, and we get a contradiction with the gap-continuity of $F$ at $\bar{x}$.

Now assume that $F$ is not gap-continuous at $\bar{x}:$ one can find some $r>0$ and some net $\left(x_{i}\right)_{i \in I}$ with limit $\bar{x}$ such that for every $i \in I$ one has $\operatorname{gap}\left(F\left(x_{i}\right), F(\bar{x})\right) \geq r$. Let

$$
C:=\{y \in Y: d(y, F(\bar{x}))<r\}
$$

so that $C$ is open, convex and disjoint from $F\left(x_{i}\right)$. The Hahn-Banach theorem yields some $y_{i}^{*} \in Y^{*}$ with norm 1 and some $t_{i} \in \mathbb{R}$ such that

$$
\sup _{y \in C}\left\langle y_{i}^{*}, y\right\rangle \leq t_{i} \leq \inf _{z \in F\left(x_{i}\right)}\left\langle y_{i}^{*}, z\right\rangle
$$

Since $\sup _{y \in C}\left\langle y_{i}^{*}, y\right\rangle=\sigma\left(y_{i}^{*}, F(\bar{x})\right)+r$ and since $\inf _{z \in F\left(x_{i}\right)}\left\langle y_{i}^{*}, z\right\rangle=-\sigma\left(-y_{i}^{*}, F\left(x_{i}\right)\right)$, we get a contradiction with relation $(2.5)$.

Remark. An alternative proof has been pointed out to us by C. Zalinescu relying on the equality $\operatorname{gap}(A, B)=d(0, A-B)$ and the following formula for the remoteness $d(0, C)$ of a nonempty convex subset $C$ of a normed vector space $Y$ :

$$
d(0, C)=\max \left\{0, \sup _{y^{*} \in S_{Y^{*}}}-\sigma_{C}\left(y^{*}\right)\right\}
$$

\section{Some properties}

The following property is obvious, but useful. It uses the excess of a subset $A$ over a subset $B$ of $Y$ defined (with the usual convention $e(\emptyset, B)=0$ for any subset $B$ and $e(A, \emptyset)=\infty$ for any nonempty subset $A$ ) by

$$
e(A, B):=\sup \{d(a, B): a \in A\} .
$$

Lemma 3.1 If $F: X \rightrightarrows Y$ is gap-continuous at $\bar{x}$ and if $G: X \rightrightarrows Y$ is another multimap such that $e(F(x), G(x)) \rightarrow 0$ as $x \rightarrow \bar{x}$, in particular if $F(x) \subset G(x)$ for every $x$ close to $\bar{x}$, then $G$ is gap-continuous at $\bar{x}$.

Here, we have used the fact that $F(x)$ is nonempty for $x$ close to $\bar{x}$, hence that $G(x)$ is nonempty too whenever $e(F(x), G(x))<+\infty$ and that $\operatorname{gap}(G(x), G(\bar{x})) \leq \operatorname{gap}(F(x), F(\bar{x}))+$ $2 e(F(x), G(x))$.

Now let us consider composition properties. 
Proposition 3.1 (a) If $F: X \rightrightarrows Y$ is gap-continuous at $\bar{x}$ and if $g: Y \rightarrow Z$ is uniformly continuous around $F(\bar{x})$, then $H:=g \circ F$ is gap-continuous at $\bar{x}$. In particular, if $F$ is gapcontinuous at $\bar{x}$, if $F(\bar{x})$ is compact and if $g$ is continuous at each point of $F(\bar{x})$, then $H:=g \circ F$ is gap-continuous at $\bar{x}$.

(b) If $F: X \rightrightarrows Y$ is l.s.c. at $(\bar{x}, \bar{y}) \in \operatorname{graph}(F)$ and if $G: Y \rightrightarrows Z$ is gap-continuous at $\bar{y}$, then $H=G \circ F$ is gap-continuous at $\bar{x}$.

Proof. (a) Our assumption on $g$ means that for any given $\varepsilon>0$ there exists $\gamma>0$ such that $d(g(y), g(z))<\varepsilon$ for all $y \in Y, z \in F(\bar{x})$ satisfying $d(y, z)<\gamma$. It is clearly satisfied $g$ is uniformly continuous on some enlargement of $F(\bar{x})$ or if $F(\bar{x})$ is compact and if $g$ is continuous at each point of $F(\bar{x})$. Since $F$ is gap-continuous at $\bar{x}$, there exists $\delta>0$ such that for all $x \in B(\bar{x}, \delta)$ there are $y(x) \in F(x), z(\bar{x}) \in F(\bar{x})$ such that $d(y(x), z(\bar{x}))<\gamma$. Thus, for every $x \in B(\bar{x}, \delta)$ one has $\operatorname{gap}(H(x), H(\bar{x}))<\varepsilon$.

(b) Given $\varepsilon>0$ let $\gamma>0$ be such that $\operatorname{gap}(G(y), G(\bar{y}))<\varepsilon$ for $y \in B(\bar{y}, \gamma)$. Now since $F$ is l.s.c. at $(\bar{x}, \bar{y})$, one can find $\beta>0$ such that $d(\bar{y}, F(x))<\gamma$ for every $x \in B(\bar{x}, \beta)$. Thus, for every $x \in B(\bar{x}, \beta)$ one can find $y \in F(x) \cap B(\bar{y}, \gamma)$ and $\operatorname{gap}(H(x), H(\bar{x})) \leq \operatorname{gap}(G(y), G(\bar{y}))<\varepsilon$.

Proposition 3.2 Let $X$ be a topological space, let $Y_{1}, \ldots Y_{m}$ be metric spaces and let $Y$ be a normed vector space.

(a) Let $F_{i}: X \rightrightarrows Y_{i}$ be gap-continuous at $\bar{x} \in X$ for $i=1, \ldots, m$. Then $F$ given by $F(x):=F_{1}(x) \times \ldots \times F_{m}(x)$ is gap-continuous at $\bar{x}$. In particular, the product of a l.s.c. multimap with an u.s.c. multimap is gap-continuous.

(b) Let $F, G: X \rightrightarrows Y$ be gap-continuous at $\bar{x} \in X$. Then $H, K$ given by $H(x):=$ $F(x)+G(x), K(x):=F(x) \cup G(x)$ are gap-continuous at $\bar{x}$. In particular the sum of a l.s.c. multifunction with an u.s.c. multifunction is gap-continuous.

(c) Let $F_{i}: X \rightrightarrows Y(i=1, \ldots, n)$ be gap-continuous at $\bar{x}$. Then $F: X \rightrightarrows Y$ given by $F(x)=\operatorname{co}\left(F_{1}(x) \cup \ldots \cup F_{n}(x)\right)$ is gap-continuous at $\bar{x}$.

Proof. The result stems from relations (2.1), (2.2), (2.3) and (2.4).

If $X$ and $Y$ are topological spaces, $X$ being compact and if $F: X \rightrightarrows Y$ is an upper semicontinuous multimap with compact values, then the image $F(X)$ of $F$ is compact. Such a property is no more valid if $F$ is just gap-continuous. In fact it is not always valid if $F$ is lower semicontinuous, as the following example shows.

Example 9. Let $X:=[0,1]$, let $Y:=\mathbb{R}$ and let $F$ be given by $F(0):=\{0\}, F(x):=\{0,1 / x\}$ for $x \in(0,1]$. Then $F$ is lower semicontinuous, but $F(X)=\{0\} \cup[1, \infty)$ which is not compact.

It is known that if $F: X \rightrightarrows Y$ is a lower semicontinuous or upper semicontinuous multimap between topological spaces and if $X$ is connected and the values of $F$ are connected, then the image $F(X)$ of $F$ is connected ([9]). With an additional assumption, such a property remains valid for gap-continuity.

Proposition 3.3 Let $X$ be a connected topological space, let $Y$ be a metric space and let $F$ : $X \rightrightarrows Y$ be gap-continuous with nonempty, compact, connected values. Then $F(X)$ is connected. 
Proof. With no loss of generality we may assume that $Y=F(X)$. Let $B, B^{\prime}$ be two open subsets of $Y$ which are disjoint and such that $Y=B \cup B^{\prime}$. Assuming they are both nonempty we will obtain a contradiction. The sets $A:=F^{-1}(B), A^{\prime}:=F^{-1}\left(B^{\prime}\right)$, where

$$
F^{-1}(B):=\{x \in X: F(x) \cap B \neq \varnothing\},
$$

are nonempty. Since for all $x \in X$ the value $F(x)$ is a connected subset of $Y, F(x)$ is contained either in $B$ or in $B^{\prime}$; hence the sets $A$ and $A^{\prime}$ are disjoint. Let us prove that $A$ and $A^{\prime}$ are open. Let $a \in A$, so that $F(a) \subset B$. Since $F(a)$ is compact, there exists some $\alpha>0$ such that $F(a)_{\alpha}:=\{y \in Y: d(y, F(a))<\alpha\}$ is contained in $B$. Since $F$ is gap-continuous at $a$, one can find a neighborhood $V$ of $a$ in $X$ such that for all $v \in V$ one has $\operatorname{gap}(F(v), F(a))<\alpha$. Thus, for all $v \in V$, there exists some $y \in F(v)$ satisfying $d(y, F(a))<\alpha$. Then $y \in B$ and, by what precedes, we have $F(v) \subset B$, hence $v \in A$. Therefore $A$ and $A^{\prime}$ are open and we get a contradiction with the connectedness of $X$.

The following proposition is a generalization of a result of Cellina [5] about approximate selections of upper semicontinuous multimaps.

Proposition 3.4 Let $F: X \rightrightarrows Y$ be a multimap from a metric space $X$ to a normed vector space $Y$ with convex values. Suppose $F$ is gap-continuous on $X$. Then, for every $\varepsilon>0$ there exists a locally Lipschitz map $f_{\varepsilon}: X \rightarrow Y$ such that for every $x \in X, f_{\varepsilon}(x) \in \operatorname{co}(F(X))$ and $d\left(f_{\varepsilon}(x), F(x)\right)<\varepsilon$.

Proof. Let $\varepsilon>0$ be given. For every $x \in X, F$ being gap-continuous at $x$, there is a neighborhood $V_{x}$ of $x$ and maps $y(\cdot, x): V_{x} \rightarrow Y, z(\cdot, x): V_{x} \rightarrow Y$ such that for all $v \in V_{x}$ one has $y(v, x) \in F(v), z(v, x) \in F(x)$ and $d(y(v, x), z(v, x))<\varepsilon$. Since $X$ is paracompact there exists a locally Lipschitz partition of unity $\left(p_{x}\right)_{x \in X}$ subordinated to the covering $\left(V_{x}\right)_{x \in X}$ of $X$. That means that $\left(p_{x}\right)_{x \in X}$ is a family of nonnegative locally Lipschitz functions summing to 1 , that their supports form a locally finite family, and that the support of $p_{x}$ is contained in $V_{x}$ for all $x \in X$. Let us set for $v \in X$

$$
f_{\varepsilon}(v):=\sum_{x \in X} p_{x}(v) z(v, x) .
$$

Then $f_{\varepsilon}$ is well defined, is locally Lipschitz and such that $f_{\varepsilon}(v) \in \operatorname{co}(F(X))$ for all $v \in X$. Since for all $v \in X$, by convexity of $F(v)$, we have

$$
\sum_{x \in X} p_{x}(v) y(v, x) \in F(v)
$$

and since $\|z(v, x)-y(v, x)\|<\varepsilon$ for all $x \in X, v \in V_{x}$, we get

$$
d\left(f_{\varepsilon}(v), F(v)\right) \leq \sum_{x \in X} p_{x}(v)\|z(v, x)-y(v, x)\| \leq \varepsilon .
$$




\section{Applications}

Given metric spaces $X, Y$, a multimap $F: X \rightrightarrows Y$ and a function $f: X \times Y \rightarrow \mathbb{R}$, numerous studies have been devoted to the study of regularity properties for the marginal function $m$ and the performance function $p$ defined by

$$
\begin{aligned}
m(x) & :=\sup \{f(x, y): y \in F(x)\}, \\
p(x) & :=\inf \{f(x, y): y \in F(x)\} .
\end{aligned}
$$

Even semicontinuity properties of such value functions are not obvious (see [3], [14] for some results and references). Here, assuming that the feasible multimap $F$ is just gap continuous, we get a coarse semicontinuity property. We suppose $f$ is uniformly continuous at $\{\bar{x}\} \times F(\bar{x})$ in the following sense: for every $\varepsilon>0$, there exists $\gamma>0$ such that $|f(x, y)-f(\bar{x}, z)| \leq \varepsilon$ for every $x \in B(\bar{x}, \gamma), z \in F(\bar{x}), y \in B(z, \gamma)$. When there exists a function $g: Y \rightarrow \mathbb{R}$ such that $f(x, y)=g(y)$ for all $(x, y) \in X \times Y$ this condition is satisfied if, and only if, $g$ is uniformly continuous around $F(\bar{x})$, as defined above.

Proposition 4.1 Suppose $F$ is gap continuous at $\bar{x} \in X$ and $f$ is uniformly continuous at $\{\bar{x}\} \times F(\bar{x})$. Then, for every $\varepsilon>0$ there exists $\delta>0$ such that for all $x \in B(\bar{x}, \delta)$ one has

$$
p(x) \leq m(\bar{x})+\varepsilon, \quad m(x) \geq p(\bar{x})-\varepsilon .
$$

When $f(\bar{x}, \cdot)$ is constant on $F(\bar{x})$, in particular when $F(\bar{x})$ is a singleton, $m$ is l.s.c. at $\bar{x}$ and $p$ is u.s.c. at $\bar{x}$.

Proof. Given $\varepsilon>0$, let $\gamma>0$ be such that $|f(x, y)-f(\bar{x}, z)| \leq \varepsilon$ for every $x \in B(\bar{x}, \gamma)$, $z \in F(\bar{x}), y \in B(z, \gamma)$. Let $\delta \in(0, \gamma]$ be such that $\operatorname{gap}(F(x), F(\bar{x}))<\gamma$ for all $x \in B(\bar{x}, \delta)$. Then, for all $x \in B(\bar{x}, \delta)$, we can find $y \in F(x), z \in F(\bar{x})$ such that $d(y, z)<\gamma$, so that

$$
\begin{aligned}
m(x) & \geq f(x, y) \geq f(\bar{x}, z)-\varepsilon \geq p(\bar{x})-\varepsilon, \\
p(x) & \leq f(x, y) \leq f(\bar{x}, z)+\varepsilon \leq m(\bar{x})+\varepsilon .
\end{aligned}
$$

When $f(\bar{x}, \cdot)$ is constant on $F(\bar{x})$, one has $m(\bar{x})=p(\bar{x})$, so that the preceding inequalities show that $m$ is l.s.c. at $\bar{x}$ and $p$ is u.s.c. at $\bar{x}$.

For the purposes of minimization of d.c. functions (see [1]), it will be useful to detect conditions ensuring that the Fenchel subdifferential of a convex function is gap-continuous. Since the subdifferential operator of a convex function is maximal monotone, such a result will be in sharp contrast with the fact proved by Gossez ([10]) that a monotone operator whose values are not singletons is never lower semicontinuous. Recall that if $f$ is a proper extendedreal valued function on a Banach space $X$, the Fenchel subdifferential of $f$ at $\bar{x} \in \operatorname{dom} f:=$ $\{x \in E: f(x)<\infty\}$ is the subset $\partial f(\bar{x})$ of the topological dual $X^{*}$ of $X$ defined by

$$
\partial f(\bar{x})=\left\{x^{*} \in X^{*}: f(x) \geq f(\bar{x})+\left\langle x^{*}, x-\bar{x}\right\rangle \forall x \in X\right\} .
$$

We first make a useful observation. 
Lemma 4.1 If $f$ is convex, if $\partial f$ is gap-continuous at $\bar{x}$, then $f$ is continuous on a neighborhood of $\bar{x}$ provided either $\partial f(\bar{x})$ is bounded or $X$ is complete and $f$ is l.s.c.

Proof. When $\partial f(\bar{x})$ is bounded and $\mathrm{f}$ is gap-continuous, one can find $r>0$, such that for each $x \in B(\bar{x}, r)$ one can find $x^{*} \in \partial f(x), \bar{x}^{*} \in \partial f(\bar{x})$ with $\left\|x^{*}-\bar{x}^{*}\right\| \leq 1$, hence $\left\|x^{*}\right\| \leq s$ for $s:=\sup \left\{\left\|\bar{x}^{*}\right\|: \bar{x}^{*} \in \partial f(\bar{x})\right\}+1$. Then for all $x \in B(\bar{x}, r)$ one has

$$
f(x) \leq f(\bar{x})-\left\langle x^{*}, \bar{x}-x\right\rangle \leq f(\bar{x})+r s .
$$

It follows that $f$ is continuous on $B(\bar{x}, r)$.

When $X$ is complete, $f$ is l.s.c. and $\partial f$ is gap-continuous, we observe that $\bar{x}$ belongs to $\operatorname{int}(\operatorname{dom} \partial f)$ which is also the interior of the domain of $f$, so that $f$ is continuous around $\bar{x}$.

Proposition 4.2 Let $F: X \rightrightarrows X^{*}$ be given by $F=\partial f$, where $f: X \rightarrow \mathbb{R} \cup\{+\infty\}$. Then

(a) If $X=\mathbb{R}^{d}$ and $f$ is convex and continuous at $\bar{x}$, then $F$ is gap-continuous at $\bar{x}$.

(b) If $f$ is convex and Fréchet differentiable at $\bar{x}$, then $F$ is gap-continuous at $\bar{x}$.

(c) If $f$ is a continuous sublinear function, then $F$ is gap-continuous at 0.

(d) If $f:=h \circ g$, where $g: X \rightarrow \mathbb{R}$ is continuous, convex with $\partial g$ gap-continuous and $h: T \rightarrow \mathbb{R}$ is convex and nondecreasing on some open interval $T$ of $\mathbb{R}$, with $g(X) \subset T$, then $F=\partial f$ is gap-continuous.

Proof. (a) It is well known that, when $X$ is finite dimensional, the subdifferential map $\partial f$ is norm-to-norm (Hausdorff) upper continuous at $\bar{x}$ (see for instance [15], Proposition 2.5.). That is, for each $\varepsilon>0$ there is $\delta>0$ such that $\partial f(x) \subset \partial f(\bar{x})+\varepsilon B^{*}$ for all $x \in B(\bar{x}, \delta)$, where $B^{*}$ is the closed unit ball of $X^{*}$. Since $f$ is bounded above on some open neighborhood of $\bar{x}$, we may suppose $f$ is subdifferentiable on $B(\bar{x}, \delta)$ and we get $\partial f(x) \cap\left(\partial f(\bar{x})+\varepsilon B^{*}\right)=\partial f(x) \neq \varnothing$ for all $x \in B(\bar{x}, \delta)$ and $\partial f$ is gap continuous at $\bar{x}$ as in Lemma 2.1 (b).

(b) Since $f$ is Fréchet differentiable at $\bar{x}$, the map $\partial f$ is norm-to-norm upper semicontinuous at $\bar{x}$ (see [15] Lemma 2.6).

(c) If $f$ is sublinear and continuous, for each $x \in X$ one has $\partial f(x)=\partial f(0) \cap H_{x}$, where $H_{x}:=\left\{x^{*} \in X^{*}:\left\langle x^{*}, x\right\rangle=f(x)\right\}$. Since $\partial f(x)$ is nonempty, one has gap $(\partial f(x), \partial f(0))=0$.

(d) In view of [7], [8], [11], [13], for every $x \in X$ we have

$$
\partial f(x)=\bigcup_{y^{*} \in \partial h(g(x))} \partial\left(y^{*} \circ g\right)(x)
$$

Now since $h$ is nondecreasing, we easily get that $y^{*} \in \mathbb{R}_{+}$for all $y^{*} \in \partial h(g(x))$, hence $\partial\left(y^{*} \circ\right.$ $g)(x)=y^{*} \partial g(x)$. Moreover, given $\bar{x} \in X$, since $g$ and $h$ are continuous at $\bar{x}$ and $g(\bar{x})$ respectively, the multimaps $\partial g$ and $\partial h$ are locally bounded around $\bar{x}$ and $\bar{y}:=g(\bar{x})$ respectively and we can find $r, s, t>0$ such that for all $x \in B(\bar{x}, r), x^{*} \in \partial g(x), y^{*} \in \partial h(g(x))$ we have $\left\|x^{*}\right\| \leq s,\left|y^{*}\right| \leq t$. If moreover $\bar{x}^{*} \in \partial g(\bar{x}), \bar{y}^{*} \in \partial h(\bar{y})$ we have

$$
\left\|\bar{y}^{*} \circ \bar{x}^{*}-y^{*} \circ x^{*}\right\| \leq\left|\bar{y}^{*}\right| \cdot\left\|\bar{x}^{*}-x^{*}\right\|+\left|\bar{y}^{*}-y^{*}\right| \cdot\left\|x^{*}\right\| \leq t\left\|\bar{x}^{*}-x^{*}\right\|+s\left|\bar{y}^{*}-y^{*}\right| .
$$

It follows that

$$
\operatorname{gap}(\partial f(x), \partial f(\bar{x})) \leq \operatorname{tgap}(\partial g(x), \partial g(\bar{x}))+\operatorname{sgap}(\partial h(g(x)), \partial h(g(\bar{x}))) \rightarrow 0 \text { as } x \rightarrow \bar{x} .
$$


The preceding result can be combined with the following one which gives another stability by composition of gap-continuity for subdifferentials.

Proposition 4.3 Let $A: X \rightarrow Y$ be a linear continuous map between two Banach spaces and let $g: Y \rightarrow \mathbb{R} \cup\{+\infty\}$ be convex l.s.c. If $\mathbb{R}_{+}(\operatorname{dom} g-A(X))$ is a closed linear subspace of $Y$ and if $\partial g$ is gap-continuous on $A(X)$ at $A(\bar{x})$, then, for $f:=g \circ A$, $\partial f$ is gap-continuous at $\bar{x}$.

Proof. Under our qualification condition, it is known that for each $x \in \operatorname{dom} f$ one has $\partial f(x)=A^{T}(\partial g(A(x)))$. Since the transpose mapping $A^{T}$ is uniformly continuous, the result is a consequence of Proposition 3.1.

Now let us deal with stability of gap continuity of subdifferentials under usual operations on the functions.

Proposition 4.4 Let $h_{1}, \ldots, h_{m}$ be a finite family of convex functions on $X$ such that $\partial h_{1}, \ldots, \partial h_{m}$ are gap-continuous at $\bar{x}$.

(a) If $h:=\max \left(h_{1}, \ldots, h_{m}\right)$, and if $h_{i}$ is continuous at $\bar{x}$ for $i=1, \ldots, m$, then $F:=\partial h$ is gap-continuous at $\bar{x}$.

(b) If $h:=h_{1} \oplus \ldots \oplus h_{m}$ is given by $h\left(x_{1}, \ldots, x_{m}\right)=h_{1}\left(x_{1}\right)+\ldots+h_{m}\left(x_{m}\right)$, then $F:=\partial h$ is gap-continuous at $\bar{x}$.

(c) If $h:=h_{1}+\ldots+h_{m}$, then $F:=\partial h$ is gap-continuous at $\bar{x}$ provided $\partial h_{i}(\bar{x})$ is bounded for $i=1, \ldots, m$, or $X$ is complete and $h_{i}$ is l.s.c. for $i=1, \ldots, m$.

Proof. (a) For $x \in X$, let $I(x):=\left\{i: h_{i}(x)=h(x)\right\}$. For $x$ close enough to $\bar{x}$, one has $I(x) \subset I(\bar{x})$, hence,

$$
\begin{aligned}
\operatorname{co}\left(\bigcup_{i \in I(x)} \partial h_{i}(\bar{x})\right) & \subset \operatorname{co}\left(\bigcup_{i \in I(\bar{x})} \partial h_{i}(\bar{x})\right)=\partial h(\bar{x}), \\
\operatorname{gap}(\partial h(\bar{x}), \partial h(x)) & \leq \operatorname{gap}\left(\operatorname{co}\left(\bigcup_{i \in I(x)} \partial h_{i}(\bar{x})\right), \operatorname{co}\left(\bigcup_{i \in I(x)} \partial h_{i}(x)\right)\right) \\
& \leq \max _{i \in I(x)} \operatorname{gap}\left(\partial h_{i}(\bar{x}), \partial h_{i}(x)\right) \leq \max _{i \in I(\bar{x})} \operatorname{gap}\left(\partial h_{i}(\bar{x}), \partial h_{i}(x)\right) \rightarrow 0 \text { as } x \rightarrow \bar{x} .
\end{aligned}
$$

(b) The result stems from Proposition 3.2 and the obvious relation

$$
\partial h\left(x_{1}, \ldots, x_{m}\right)=\partial h_{1}\left(x_{1}\right) \times \ldots \times \partial h_{m}\left(x_{m}\right) .
$$

(c) In both cases, $h_{i}$ is continuous around $\bar{x}$ by Lemma 4.1 . Thus $\partial h(x)=\partial h_{1}(x)+\ldots+\partial h_{m}(x)$ and the result is a consequence relation (2.2). When $X$ is complete and for all $i$, the function $h_{i}$ is l.s.c. one can also apply assertion (b) and the preceding proposition with $Y:=X^{m}$, $A(x):=(x, \ldots, x)$ since $\bar{x}$ belongs to the interiors of the domains of $h_{i}$.

Now, let us turn to the nonconvex case. Recall that the Fréchet subdifferential of a function $f: X \rightarrow \mathbb{R} \cup\{+\infty\}$ at a point $\bar{x} \in \operatorname{dom} f$ is defined by 


$$
\partial^{F} f(\bar{x})=\left\{x^{*} \in E^{*}: \liminf _{\|v\| \rightarrow 0} \frac{f(\bar{x}+v)-f(\bar{x})-\left\langle x^{*}, v\right\rangle}{\|v\|} \geq 0\right\}
$$

and $\partial^{F} f(\bar{x})=\varnothing$ if $\bar{x} \notin \operatorname{dom} f$.

Proposition 4.5 Let $h:=k \circ j$, where $j: X \rightarrow Y$ is of class $C^{1}$ around $\bar{x}$ and $k: Y \rightarrow \mathbb{R}$ is such that $\partial^{F} k$ is gap-continuous at $\bar{y}:=j(\bar{x})$ and $\partial^{F} k(\bar{y})$ is bounded. Then $\partial^{F} h$ is gapcontinuous at $\bar{x}$.

Proof. The result stems from the inclusion $\partial^{F} k(j(x)) \circ j^{\prime}(x) \subset \partial^{F} h(x)$ and from the fact that there exist maps $y^{*}, \bar{y}^{*}$ defined on a neighborhood $V$ of $\bar{y}$ such that $y^{*}(v) \in \partial^{F} k(v), \bar{y}^{*}(v) \in$ $\partial^{F} k(\bar{y})$ for all $v \in V$ and $\left\|y^{*}(v)-\bar{y}^{*}(v)\right\| \rightarrow 0$ as $v \rightarrow \bar{y}$, so that, $y^{*}(j(x)) \circ j^{\prime}(x) \in \partial^{F} h(x)$, $\bar{y}^{*}(j(x)) \circ j^{\prime}(\bar{x}) \in \partial^{F} h(\bar{x})$ and

$$
\begin{aligned}
& \left\|y^{*}(j(x)) \circ j^{\prime}(x)-\bar{y}^{*}(j(x)) \circ j^{\prime}(\bar{x})\right\| \\
& \leq\left\|\left(y^{*}(j(x))-\bar{y}^{*}(j(x))\right) \circ j^{\prime}(x)\right\|+\left\|\bar{y}^{*}(j(x)) \circ\left(j^{\prime}(x)-j^{\prime}(\bar{x})\right)\right\| \rightarrow 0 \text { as } x \rightarrow \bar{x} .
\end{aligned}
$$

Acknowlegments. We are grateful to C. Zălinescu for his remarks on an early version of the manuscript.

\section{References}

[1] T. Amahroq, J.-P. Penot and A. Syam, Subdifferentiation and minimization of the difference of two functions, submitted.

[2] Aubin, J.-P. and H. Frankowska, Set-Valued Analysis, Birkhäuser, Basel (1990).

[3] Bank, B., Guddat, J., Klatte, D., Kummer, B. and Tammer, K., Non-linear parametric optimization, Birkhäuser Verlag Basel (1983).

[4] Beer, G., Topologies on Closed and Closed Convex Sets, Mathematics and its Applications 268, Kluwer, Dordrecht (1993).

[5] Cellina, A., A theorem on the approximation of set-valued mappings, Rend. Acad. Nac. Lincei 47, 429-433 (1969).

[6] Champion, T., Duality gap in convex programming, Math. Program. 99, No. 3 (A), (2004), 487-498.

[7] Combari, C., Laghdir, M.and Thibault, L., A note on subdifferentials of convex composite functionals, Arch. Math. 67, No.3, 239-252 (1996).

[8] Combari, C., Laghdir, M. and Thibault, L., On subdifferential calculus for convex functions defined on locally convex spaces, Ann. Sci. Math. Qué. 23, No.1, 23-36 (1999). 
[9] Correa, R., Hiriart-Urruty, J.-B. and Penot, J.-P., A note on connected set-valued mappings, Boll. Unione Mat. Ital., VI. Ser., C, Anal. Funz. Appl. 5, 357-366 (1986).

[10] Gossez, J.-P., A note on multivalued monotone operators, Mich. Math. J. 17, 347-350 (1970).

[11] Hiriart-Urruty, J.-B., Gradients généralisés de fonctions composées. Applications. C. R. Acad. Sci., Paris, Sér. A 285, 781-784 (1977).

[12] Holmes R.B., Geometric Functional Analysis and its Applications, Guaduate Texts in Mathematics 24, Springer Verlag, New York (1975).

[13] Lemaire, B., Application of a subdifferential of a convex composite functional to optimal control in variational inequalities, in: Nondifferentiable optimization: motivations and applications, Proc. IIASA Workshop, Sopron/Hung. 1984, Lect. Notes Econ. Math. Syst. 255, 103-117 (1985).

[14] Penot, J.-P., Calmness and stability properties of marginal and performance functions, Numerical Functional Analysis and Optimization, 25 (3-4), 287-308 (2004).

[15] Phelps, R., Convex functions, Monotone Operators and Differentiability, Lecture Notes in Mathematics, Springer Verlag, Berlin, Heidelberg, New York 1989.

[16] Rockafellar, R.T., Wets R. J.-B., Variational Analysis, Grundlehren der Mathematischen Wissenschaften, Vol. 317, Springer-Verlag, Berlin (2002).

[17] Zălinescu, C., Convex Analysis in General Vector Spaces, World Scientific, Singapore, (2002). 